
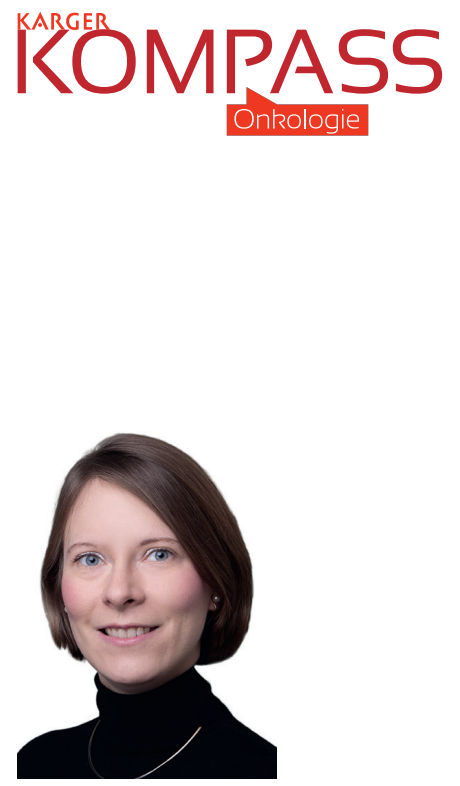

Nina Rosa Neuendorff

Koordination «OnkoCampus»

\title{
Wenn Onkologie und Rheumatologie aufeinandertreffen
}

Liebe Leserinnen und Leser,

herzlich willkommen! Wir hoffen, Ihnen auch in der aktuellen Ausgabe wieder interessante Themen präsentieren zu dürfen!

Diesmal widmet sich die vorliegende Ausgabe den Überschneidungsgebieten der Onkologie und Rheumatologie. Die Einführung der Checkpoint-Inhibitoren vor wenigen Jahren hat die Onkologie grundlegend revolutioniert und die Therapiemöglichkeiten vieler schwer zu behandelnder Malignome deutlich verbessert. Doch die immunvermittelten Nebenwirkungen stellen teilweise vollkommenes Neuland in der Onkologie dar, beispielsweise die Hypophysitis, die vor der Einführung der Checkpoint-Inhibitoren eine vollkommene Rarität war. Dementsprechend besteht eine dringende Notwendigkeit, sich immer wieder mit diesen immunvermittelten Nebenwirkungen zu befassen. Selten, aber sehr schwerwiegend können renale Toxizitäten verlaufen. Dr. Sophie Anker und Dr. Liya Hannemann geben einen Überblick über diese seltene Nebenwirkung und den Umgang damit. Malignome und rheumatologische Erkrankungen interagieren auf vielen Ebenen, was ausgesprochen schlecht erforscht ist. Aus diesem Grund wurde 2018 das MalheuR-Projekt ins Leben gerufen. Dr. Karolina Benesova und Dr. Jan Leipe stellen das Projekt vor.

Mit der vorliegenden Aufgabe des Kompass Onkologie beende ich meine Tätigkeit als Campus Koordinatorin. Übernehmen wird diese Aufgabe ab dem kommenden Jahr meine Kollegin, Dr. Maike Janssen, die mit vielen neuen Ideen frischen Wind in den Campus bringen wird. Mir hat die Gestaltung der Campus-Sektion und die Zusammenarbeit mit der Redaktion immer sehr viel Spaß gemacht!

Nun wünsche ich Ihnen viel Spaß beim Lesen und scheuen Sie sich nicht, uns bei Anregungen oder Kritik zu kontaktieren, vielleicht gibt es ja auch Themenwünsche?

Herzliche Grüße

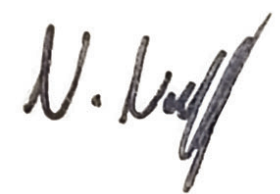

Nina Rosa Neuendorff

\section{KARGER}

Fax +497614520714

information@karger.com

www.karger.com
() 2019 S. Karger GmbH, Freiburg
Medizinische Klinik V (Hämatologie, Onkologie und Rheumatologie)

NinaRosa.Neuendorff@med.uni-heidelberg.de 


\section{Aus der Praxis}

\section{Das Heidelberger MalheuR-Register: Maligne und rheumatische Erkrankungen - zwei Extreme des fehlgesteuerten Immunsystems}

Die internistische Rheumatologie umfasst etwa 400 entzündlichrheumatische Erkrankungen autoimmuner oder autoinflammatorischer Genese, die etwa 2\% der erwachsenen Bevölkerung in Deutschland betreffen. Wie kaum ein anderer Fachbereich hat die Rheumatologie in den letzten Jahren von wissenschaftlicher Forschung und der Zulassung innovativer Medikamente profitiert Dadurch hat sich nicht nur die Funktionalität der Patienten erheblich verbessert (weniger Arbeitsunfähigkeit/Erwerbsminderung und körperliche Behinderung/Pflegebedürftigkeit), sondern ist auch die Lebenserwartung signifikant gestiegen. Sowohl die rheumatische Grunderkrankung als auch immunmodulatorische bzw. -suppressive Therapien stellen jedoch Risikofaktoren für verschiedene Begleit- und Folgeerkrankungen dar. Besondere Relevanz haben in diesem Zusammenhang maligne Erkrankungen, deren Inzidenz- und Prävalenzraten in der Allgemeinbevölkerung insbesondere aufgrund der demografischen Entwicklung stetig zunehmen. Gleichzeitig ist auch im Fachgebiet der Onkologie durch klinische und experimentelle Fortschritte ein Rückgang der Malignom-assoziierten Sterblichkeitsrate zu verzeichnen, sodass die Anzahl an Langzeitüberlebenden nach Malignomdiagnose zunimmt.

Aufgrund der zunehmenden Prävalenz maligner und entzündlichrheumatischer Erkrankungen ist die Wahrscheinlichkeit für eine Koinzidenz beider Krankheitsentitäten in einem Individuum entsprechend höher. Allerdings sind die Zusammenhänge und Wechselwirkungen auf klinischer und molekulargenetischer Ebene bislang nur in Ansätzen erforscht. Der Mangel an einerseits verlässlichen Daten zum krankheits- und therapieassoziierten Malignom(rezidiv) risiko bei bestimmten entzündlich-rheumatischen Erkrankungen und andererseits an standardisierten Handlungsempfehlungen bei Koinzidenz beider Krankheitsentitäten verunsichert im klinischen Alltag sowohl die Patienten als auch behandelnde Ärzte. In der Folge werden rheumatische Symptome bei Patienten mit Malignomen regelhaft untertherapiert, unter der Vorstellung, mögliche negative Effekte auf die maligne Erkrankung (Tumorprogress bzw. Verlust des Ansprechens auf Tumortherapie etc.) zu vermeiden. Um diese Lücken zu schließen, wurde das MalheuR-Projekt (Akronym aus malignen und rheumatischen Erkrankungen) als Registerstudie von der Sektion Rheumatologie des Universitätsklinikums Heidelberg 2018 initiiert. Die Koinzidenz von entzündlich-rheumatischen Erkrankungen und Malignomen wird dabei aus 3 verschiedenen Dimensionen in Subregistern betrachtet, die im Weiteren näher dargestellt werden

\section{Rheumatologische Patienten mit Malignom}

(RheuMal-Register)

Wenn Patienten mit entzündlich-rheumatischen Erkrankungen ein Malignom entwickeln, ist sowohl ein Zusammenhang mit der rheumatischen Grunderkrankung (z.B. erhöhtes Lymphomrisiko beim Sjögren-Syndrom) als auch mit der durchgeführten Therapie zu diskutieren (z.B. erhöhte Risiken für Urothelkarzinome nach Cyclophosphamid, für Hautmalignome nach Azathioprin oder nach therapeutischer UV-Bestrahlung (PUVA) der Psoriasis bei Psoriasis-Arthritis). Für viele medikamentöse Therapien sind die Zusammenhänge nicht endgültig geklärt. Die Risikobeurteilung wird zusätzlich durch teilweise erst kurze Zulassungsdauer und damit geringe Erfahrung einerseits sowie kombinierte bzw. sequentielle Anwendung verschiedener Basistherapeutika andererseits erschwert.

Entsprechend groß ist die Verunsicherung bezüglich des Malignomrisikos, insbesondere bei Therapien, die an zentralen Schaltstellen des Immunsystems eingreifen. Die landesweiten Register leisten zwar einen wesentlichen Beitrag bei der Langzeitbeobachtung und Erfassung seltener Risiken bestimmter Therapien, jedoch fehlen bisher größtenteils krankheits- und therapieübergreifende Korrelationen der Patientencharakteristika, der rheumatischen und malignen Diagnosen, der Krankheitsverläufe und -Outcomes und ein Vergleich zur rheumatisch nicht vorbelasteten Allgemeinbevölkerung.

Hier setzt das RheuMal-Register an, das bereits erste Hinweise erbringen konnte, dass Patienten mit entzündlich-rheumatischen Erkrankungen früher an bestimmten Malignomen erkranken können als die gesunde Vergleichspopulation. Zusätzlich soll die Datenlage zum klinischen Management der entzündlich-rheumatischen Erkrankung und dem Rezidivrisiko nach der Diagnose des Malignoms gestärkt werden.

\section{Paraneoplastische Rheumatische Symptome bei Malignom-} patienten (ParaRheuMa-Register)

Unspezifische muskuloskelettale Symptome und Funktionseinschränkungen werden zumindest zeitweise bei etwa einem Viertel der Allgemeinbevölkerung beobachtet, stellen demnach häufige Ereignisse dar und werden daher regelhaft als mögliches Leitsymptom einer malignen Erkrankung nicht ernst genommen. Paraneoplastische Symptome werden infolgedessen häufig erst verzögert identifiziert, in aller Regel durch den atypischen, therapierefraktären Verlauf einer vermeintlichen entzündlich-rheumatischen Erkrankung, mit konsekutiver Diagnose der malignen Erkrankung. Charakteristisch für eine Paraneoplasie ist dabei nicht nur die zeitliche Assoziation (synchrones, allenfalls wenige Monate auseinanderliegendes Auftreten der Symptome), sondern auch die Beobachtung, dass die Symptome häufig nur durch die erfolgreiche Malignomtherapie nachhaltig positiv beeinflusst werden. Im Rahmen des ParaRheuMa-Registers soll insbesondere erforscht werden, ob einerseits bestimmte Symptomkomplexe gehäuft paraneoplastisch auftreten und andererseits Zusammenhänge zwischen paraneoplastischen muskuloskelettalen Symptomen und bestimmten Neoplasien existieren. 
Therapieinduzierte Rheumatische Symptome bei Malignompatienten (TRheuMa-Register)

Neue Aufmerksamkeit erlangten die wechselseitigen Beziehungen zwischen entzündlich-rheumatischen und malignen Erkrankungen durch das Auftreten rheumatischer Symptome als Nebenwirkungen der Immuntherapie mit Checkpoint-Inhibitoren (sogenannte «immune-related adverse events» (irAEs)). Der Wirkmechanismus dieser Substanzen besteht v.a. in der Blockierung der über Checkpoint-Rezeptoren vermittelten Escapemechanismen von Tumorzellen mit erneuter Aktivierung der physiologischen Tumorclearance durch zytotoxische T-Zellaktivität. Hierbei wird jedoch eine überschießende Immunantwort mit reduzierter Toleranz gegenüber körpereigenen Zellen ausgelöst: irAEs können alle Organsysteme betreffen und klinisch bekannten Autoimmunopathien ähneln. So können auch alle Krankheitsbilder des rheumatischen Formenkreises nachgeahmt werden, wobei Arthralgien und/oder Myalgien als milde irAEs häufig auftreten und in klinischen Studien von 10-20\% der Patienten berichtet wurden. Da häufig typische serologische (Autoantikörper) Befunde «klassischer» Erkrankungen fehlen, ergibt sich derzeit meist nur aus dem Verlauf, ob es sich um eine immunvermittelte entzündliche Nebenwirkung oder eine therapiegetriggerte Erstmanifestation einer tatsächlichen entzündlich-rheumatischen Erkrankung handelt. Obgleich rheumatische irAEs selten vital gefährdend sind, können sie dennoch erhebliche Schmerzen und Funktions-, bzw. Mobilitätseinschränkungen verursachen, die entsprechender Behandlung bedürfen. Besondere Relevanz haben die rheumatischen irAEs dahingehend, dass betroffene Patienten ein besseres Tumoransprechen und Langzeitüberleben aufweisen. Dieser Vorteil soll möglichst nicht durch eine immunmodulierende/-supprimierende Therapie beeinträchtigt werden.

Die Ziele des TRheuMa-Registers sind, aus der rheumatologischen Perspektive erstmals prospektiv eine systematische Übersicht zu Häufigkeit, Art und Verlauf muskuloskelettaler Nebenwirkungen unter antineoplastischen Therapien zu erstellen. Besonderer Fokus sind hierbei zwar die irAEs unter Checkpoint-Inhibition, prinzipiell können aber auch andere antineoplastische Therapien wie beispielsweise Aromatasehemmer bei Malignompatienten rheumatische Symptome verursachen.

\section{Forschungsagenda}

Die weitere Erforschung beider Krankheitsentitäten im Rahmen des MalheuR-Registers bietet die einzigartige Möglichkeit, zwei Extreme des fehlgeleiteten Immunsystems auch auf der grundlagenwissenschaftlichen Ebene näher zu charakterisieren. Die Neoplasie Entstehung mit verminderter Tumorsurveillance und pathologisch reduzierter Immunantwort auf der einen Seite sowie die Autoimmunopathie-Entstehung mit autoaggressiver Immunzellaktivität als Gegenpol bieten wertvolle Ansatzpunkte für weitere Forschungsarbeit: Durch Verknüpfung des Registers mit bestehenden Biobank-Projekten können Hinweise auf Pathogenese beider Entitäten gewonnen und potenziell neue Biomarker und Therapietargets identifiziert werden.

\section{Fazit}

Die bisher nicht dagewesene Betrachtung der Zusammenhänge und Wechselwirkungen entzündlich-rheumatischer und maligner Erkrankungen aus 3 Blickwinkeln zeichnet das MalheuR-Register aus. Durch die Erforschung der Schnittstellen zwischen beiden Krankheitsentitäten basierend auf demographischen Daten, Latenzzeiterfassung der Charakteristika, therapeutischen Managements und Outcomes sowie der Korrelationen spezifischer Diagnosen und Therapien kann ein Wissenszuwachs generiert werden, der betroffenen Patienten auf mehreren Ebenen zugutekommt und bestehende Unsicherheiten im klinischen Alltag mindern kann.

\section{Kontakt}

Dr. Karolina Benesova, Universitätsklinik Heidelberg, Innere Medizin V, Sektion Rheumatologie, Heidelberg; Dr. Jan Leipe, Universitätsklinik Mannheim, V. Medizinische Klinik, Sektion Rheumatologie, Mannheim

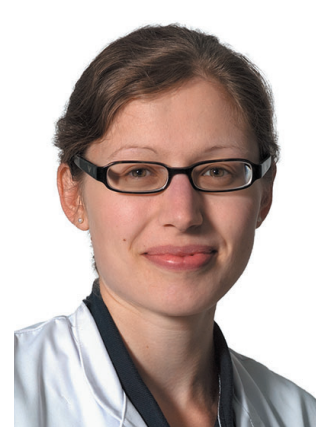

Dr. Karolina Benesova

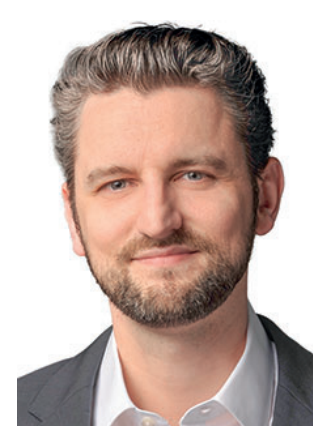

Dr. Jan Leipe

\section{Literatur}

1 Benesova K, Lorenz H-M, Leipe J, et al. How I treat cancer: treatment of rheumatological side effects of immunotherapy. ESMO Open 2019;4.

2 Buder-Bakhaya K, Benesova K, Schulz C, et al. Characterization of arthralgia induced by $\mathrm{PD}-1$ antibody treatment in patients with metastasized cutaneous malignancies. Cancer Immunol Immunother 2018;67:175-82.

3 Leipe J, Christ LA, Arnoldi AP, et al. Characteristics and treatment of new-onset arthritis after checkpoint inhibitor therapy. RMD Open 2018;4:e000714.

4 Zink A, Albrecht K, Wie häufig sind muskuloskeletale Erkrankungen in Deutschland? Z Rheumatol 2016, 75:346-353. 


\section{Aus der Praxis}

\section{Renale Nebenwirkungen von Immun-Checkpoint-Inhibitoren}

Die Neuentwicklung von Immun-Checkpoint-Inhibitoren hat die Therapie vieler Krebsentitäten grundlegend verändert. Auf der einen Seite eröffnet der neue Wirkmechanismus auch neue Therapieoptionen, auf der anderen Seite ergibt sich jedoch ein neues Spektrum an unerwünschten Nebenwirkungen. So kommt es bei der Therapie mit Immun-Checkpoint-Inhibitoren vermehrt zu immunologisch vermittelten Toxizitäten, die im gesamten Organismus auftreten können. In diesem Artikel möchten wir eine Zusammenfassung über die renalen Nebenwirkungen von ImmunCheckpoint-Inhibitoren und den Umgang mit ihnen geben.

\section{Immun-Checkpoint-Inhibitoren: Wirkweise und Anwendungsgebiete}

Tumorzellen können sich auf verschiedenen Wegen dem Immunsystem entziehen, wobei man vom sogenannten «Immun-Escape» spricht. Beispielsweise exprimieren Tumorzellen vermehrt Oberflächenproteine, welche die Aktivierung des Immunsystems gegen die Tumorzelle verhindern. So entsteht, u.a. durch die Aktivierung von Immun-Checkpoint-Molekülen, zu denen das zytotoxische TLymphozyten-Antigen 4 (CTLA-4), das programmierte Zelltod Protein 1 (PD-1) und der programmierte Zelltod Protein Ligand 1 (PDL1) zählen, ein immunsuppressives Tumormikromilieu [1]. An diesem Mechanismus setzen die Immun-Checkpoint-Inhibitoren (ICI) an. Sie binden CTLA-4, PD-1 oder PD-L1 und ermöglichen durch diese Inhibition eine gegen die Tumorzellen gerichtete Aktivierung des Immunsystems. Eingesetzt werden Immun-Checkpoint-Inhibitoren $(\mathrm{ICl})$ aktuell insbesondere beim metastasierten Bronchialkarzinom, dem metastasierten Nierenzellkarzinom sowie dem Malignen Melanom. Tabelle 1 gibt einen Überblick über die Erkrankungen, bei denen $\mathrm{ICl}$ als Therapie zugelassen sind.

Table 1. Zugelassene Indikationen verschiedener ICI

\begin{tabular}{ll}
\hline Substanz & Zugelassene Einsatzgebiete \\
\hline $\begin{array}{ll}\text { CTLA4-Antagonisten } \\
\text { Ipilimumab }\end{array}$ & $\begin{array}{l}\text { Melanom, Nierenzellkarzinom (beide jeweils auch } \\
\text { in Kombination mit Nivolumab) }\end{array}$ \\
\hline $\begin{array}{ll}\text { Anti-PD-1-Antikörper } \\
\text { Nivolumab }\end{array}$ & Melanom, Nierenzellkarzinom, NSCLC, Hodgkin- \\
\hline Pembrolizumab & Melanom, NSCLC, Hodgkin-Lymphom, PEKa \\
\hline $\begin{array}{ll}\text { Anti-PDL1-Antikörper } \\
\text { Avelumab }\end{array}$ & Merkelzellkarzinom \\
\hline Durvalumab & NSCLC \\
\hline Atezolizumab & NSCLC, Urothelkarzinom \\
\hline NSCLC, non-small cell lung cancer; PEKa, Plattenepithelkarzinom der Kopf- \\
Hals Region.
\end{tabular}

\section{Immunologisch vermittelte Toxizitäten durch ICI}

Insgesamt kommt es bei etwa 60\% der Patienten mit ICI-Therapie zu immunologisch vermittelten Toxizitäten (immun-related adverse events, irAEs), wenn man alle Schweregrade zusammenfasst [2]. IrAEs können jedes Organ und jedes Gewebe betreffen und sowohl während als auch nach der Therapie auftreten. Dabei variieren Inzidenz, Manifestationsort und -zeitpunkt in Abhängigkeit der ICl-Klasse. Beispielsweise kommt es bei CTLA-4-Blockern häufiger zu einer Hypophysitis und Kolitis, während unter PD-1-Blockade vermehrt Thyreoiditiden, Arthritiden und Pneumonitiden auftreten [3]. Des Weiteren treten Endokrinopathien und Dermatitiden typischerweise zeitnah nach Therapiebeginn auf [3]. Als pathophysiologiche Ursache werden eine direkte Antikörper-vermittelte Toxizität, eine Immunkomplex-vermittelte Toxizität, eine zellvermittelte Toxizität sowie eine Zytokin-vermittelte Toxizität diskutiert [1].

\section{Immunologisch vermittelte renale Nebenwirkungen (irAEs)}

Renale irAEs gehören zu den selteneren irAEs. In einer erst kürzlich veröffentlichen Metaanalyse über PD-1-Inhibitoren kam es in 2,2\% der Fälle zu einem akuten Nierenversagen (ANV). Bei CTLA-4-Inhibitoren sowie Kombinationstherapien ist die Inzidenz etwas höher [1]. Es konnte jedoch nicht nachgewiesen werden, dass das ANV immer ausschließlich durch die ICl-Therapie ausgelöst wurde. Die klinische Hauptmanifestation ist das ANV mit einem Anstieg der Retentionsparameter. Weiterhin kann eine Proteinurie, Hämaturie oder Elektrolytveränderung auftreten. Renale irAEs sind zu jedem Zeitpunkt während und nach der Therapie beschrieben [1]. Die Schweregradeinteilung der renalen irAEs erfolgt gemäß der Common Terminology Criteria for Adverse Events (CTCAE, Version 5), welche sich ausschließlich nach dem Anstieg des Serumkreatinins richtet (Einteilung siehe Tabelle 2). In etwa einem Drittel der Fälle kommt es zu einer höhergradigen Komplikation ('III/IV) [1]. Obwohl sich die renalen irAEs klinisch und laborchemisch ähneln, ist das histologische Spektrum ausgesprochen breit. Am häufigsten wird eine akute (tubulo-) interstitielle Nephritis (A(T)IN), z.T. mit Nachweis von Granulomen, beschrieben. Seltener handelt es sich um glomeruläre Erkrankungen, wie z.B. eine Minimal Change-Glomerulonephritis (MCG) oder eine fokal segmentale Glomerulosklerose (FSGS). In vereinzelten Fällen wurden auch eine thrombotische Mikroangiopathie (TMA), eine membranoproliferative Glomerulonephritis (MPGN) oder ein Goodpasture-Syndrom beschrieben. Es scheint, als könnten viele der bekannten immunologisch vermittelten Pathologien an der Niere auch durch ICI bzw. durch die von ihnen verstärkte Immunreaktion ausgelöst werden. Differentialdiagnostisch ist wichtig zu bedenken, dass die A(T)IN jedoch deutlich häufiger im Rahmen einer Typ-IV-Hypersensitivitätsreaktion auf bestimmte Medikamente (Protonenpumpeninhibitoren, NSAR, Diuretika, einige Antibiotika) auftritt. Seltener kann sie auch durch eine Infektion, z.B. mit Legionellen, oder durch Autoimmunphänomene 
Table 2. Management renaler irAEs

\begin{tabular}{|c|c|c|}
\hline Schweregrad & Management & Diagnostik \\
\hline $\begin{array}{l}\text { I }^{\circ}(\text { Kreatinin } 1,5 \times \text { AW oder } \\
>1-1,5 \times \text { ONW })\end{array}$ & $\begin{array}{l}\text { Therapiefortführung unter regelmäßiger Kontrolle der } \\
\text { Retentionsparameter ggf. Pausierung nephrotoxischer Medikamente }\end{array}$ & \multirow{4}{*}{$\begin{array}{l}\text { Ausschluss möglicher } \\
\text { Differentialdiagnosen (prä-/intra-/ } \\
\text { postrenales ANV): } \\
\text { Volumenstatus? HWI? Nephrotoxische } \\
\text { Medikation? Postrenale Obstruktion? } \\
\text { Evaluation Nierenbiopsie ab } \\
\text { Schweregrad II }\end{array}$} \\
\hline $\begin{array}{l}\boldsymbol{I I}^{\circ} \text { (Kreatinin }>1,5-3 \times \text { AW oder } \\
>1,5-3 \times \text { ONW })\end{array}$ & $\begin{array}{l}\text { Therapiepause und orale Glukokortikoid-Therapie } \\
\text { (Prednisolon 0,5-1 mg/kg); Evaluation Wiederaufnahme der Therapie } \\
\text { bei rückläufigen Retentionsparametern }\end{array}$ & \\
\hline $\begin{array}{l}\text { III' }^{\circ} \text { (Kreatinin }>3 \times \text { AW oder } \\
>3-6 \times \text { ONW })\end{array}$ & $\begin{array}{l}\text { Therapieabbruch und orale Glukokortikoid-Therapie } \\
\text { (Prednisolon } 0,5-1 \mathrm{mg} / \mathrm{kg} \text { ) }\end{array}$ & \\
\hline IV $^{\circ}$ (Kreatinin >6 × ONW) & $\begin{array}{l}\text { Therapieabbruch und orale Glukokortikoid-Therapie } \\
\text { (Prednisolon 0,5-1 mg/kg); Stationäre Aufnahme; ggf. Hämodialyse }\end{array}$ & \\
\hline
\end{tabular}

Zusammenfassung der Handlungsempfehlungen sowie diagnostischen Schritte bei Auftreten renaler irAEs in Anlehnung an die ESMO Guidelines [2]. Schweregrade der renalen irAEs in Anlehnung an Common Terminology Criteria for Adverse Events (CTCAE), Version 5.0, November 2017, National Institutes of Health, National Cancer Institute.

ausgelöst werden. In den von uns untersuchten Fällen mit $A(T) I N$ bestand in über 50\% der Fälle eine Komedikation mit den oben genannten Medikamenten. Daraus resultiert die Hypothese, dass die ICl-Therapie die Toleranz des Immunsystems gegenüber bestimmten Medikamenten herabsetzt, woraufhin sich eine $A(T) I N$ entwickelt. Hierzu passt, dass sich die ICl-induzierte A(T)IN histologisch nicht von der Medikamenten-induzierten A(T)IN unterscheidet [4]. Bei Verdacht auf ein renales irAE ist somit eine Medikamentenanamnese sowie die Pausierung nephrotoxischer Medikamente von zentraler Bedeutung.

Gegenwärtig erhalten viele Patienten ICl erst nach Versagen mehrerer Therapielinien bzw. bei chemotherapierefraktärer Erkrankung, sodass eine unnötige Therapiepausierung bzw. ein Therapieabbruch unbedingt vermieden werden sollte. Essentiell hierfür sind eine schnelle und sichere Diagnosefindung. Dabei müssen die in Frage kommenden Differentialdiagnosen für ein ANV ausgeschlossen werden. Dazu zählen das prä- und postrenale Nierenversagen sowie eine Einschränkung der Nierenfunktion durch andere nephrotoxische Medikamente oder ein Tumorlysesyndrom. Wird nach Ausschluss der Differentialdiagnosen von einem ANV durch $\mathrm{ICl}$ ausgegangen, so ist ab einem Schweregrad von II eine Nierenbiopsie zu erwägen, um eine präzise Diagnose stellen und eine entsprechende Therapie einleiten zu können. Aktuell aufkommende Therapieschemata mit einer standardisierten Kombination aus $\mathrm{ICl}$ und platinhaltiger Chemotherapie werden die Differentialdiagnostik noch weiter erschweren, da Platine bekanntermaßen einen akuten Tubulusschaden auslösen können. Die Steroidresponsivität renaler irAEs liegt ungefähr bei $80 \%$ und ist somit recht hoch [1]. In Hinblick auf die Wirksamkeit der IClTherapie wurde jedoch kürzlich gezeigt, dass diese durch eine frühe, parallel zur ICl-Therapie erfolgte Steroidgaben reduziert sein kann [5]. Dennoch ist eine Immunsuppression bei schweren Verläufen renaler irAEs nach Risiko-Nutzen-Abwägung meist indiziert.

Bezüglich einer Re-Exposition im Falle eines Rückgangs der irAEs unter Steroidtherapie gibt es bisher wenige Daten, sodass es sich letzten Endes um eine individuelle Entscheidung handelt.

\section{Zusammenfassung}

Renale irAEs sind selten, ihre Inzidenz wird jedoch vermutlich unterschätzt. Histologisch wird meist eine A(T)IN diagnostiziert. Bei Verdacht auf ein irAE sollten Differentialdiagnosen eines ANV ausgeschlossen werden. Eine Nierenbiopsie sollte aufgrund der möglichen Therapiekonsequenz ab einer Toxizität II erwogen werden. Unter Steroiden und Therapiepausierung/-stopp ist das ANV meist reversibel. Die Entscheidung in Hinblick auf eine Re-Exposition bzw. einen ICl-Klassenwechsel bleibt eine Einzellfallentscheidung.

\section{Disclosure Statement}

Beide Autoren geben keine Interessenkonflikte bezüglich des vorliegenden Artikels an.

Kontakt: Liya Hannemann, Sophie Anker: Medizinische Klinik V (Hämatologie, Onkologie und Rheumatologie), Universitätsklinik Heidelberg

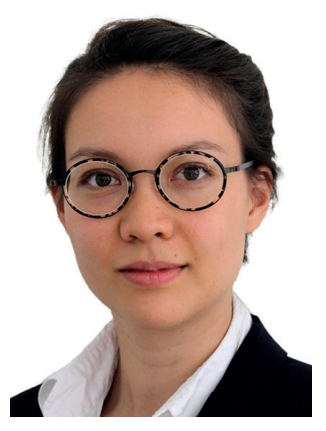

Liya Hannemann

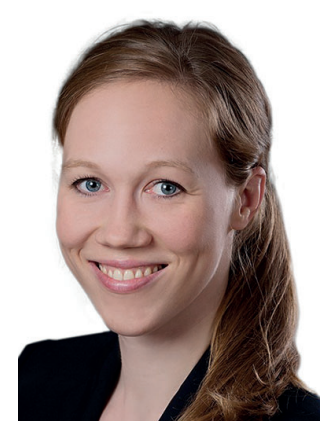

Sophie Anker

\section{Literaturverzeichnis}

1 Sury, K., M.A. Perazella, and A.C. Shirali, Cardiorenal complications of immune checkpoint inhibitors. Nat Rev Nephrol, 2018. 14(9): p. 571-588.

2 Haanen, J., et al., Management of toxicities from immunotherapy: ESMO Clinical Practice Guidelines for diagnosis, treatment and follow-up. Ann Oncol, 2017. 28(suppl_4): p. iv119-iv142.

3 Kim, S.T. and M.E. Suarez-Almazor, Managing immune dysregulation resulting from immune checkpoint inhibitors: impact of the ASCO guidelines and key takehomes for immunologists. Expert Rev Clin Immunol, 2019. 15(3): p. 211-213.

4 Murakami, N., S. Motwani, and L.V. Riella, Renal complications of immune checkpoint blockade. Curr Probl Cancer, 2017. 41(2): p. 100-110.

5 Fuca, G., et al., Modulation of peripheral blood immune cells by early use of steroids and its association with clinical outcomes in patients with metastatic non-small cell lung cancer treated with immune checkpoint inhibitors. ESMO Open, 2019. 4(1): p. e000457. 